\author{
ANNALES \\ POLONICI MATHEMATICI \\ XXIX (1974)
}

\title{
Sur un système d'équations aux différences finies
}

\author{
par Zygmunt Butlewski (Poznań)
}

Résumé. On considére le système d'équations aux différences finies

(*)

$$
\Delta y=h A(x) \varepsilon, \quad \Delta z=h B(x) y,
$$

où les coefficients $A(x), B(x)$ sont des fonctions continues dans l'intervalle $J$ et 1 s distance $h$ est une constante positive. Soit $y(x), z(x)$ une solution continue et oscillante du système (*) pour $x \in J$. On suppose que les inégalités suivantes sont remplies:

$\left(\begin{array}{c}* \\ *\end{array}\right)$

$$
0<A_{1}<A(x), \quad B(x) \leqslant-B_{1}<0 \quad\left(A_{1}, B_{1}=\text { Const }\right), \quad x \in J .
$$

Dans ces conditions on démontre que la longueur de tout intervalle entre deux zéros consécutifo de la fonction $y(x)$ (resp. $z(x)$ ) ne dépasse pas $2 h(2+1 / p)$, où l'on a $0<p=h^{2} A_{1} B_{1}<2$.

On obtient aussi le résultat suivant: tout sous-intervalle de $J$ dont la longueur dépasse $\lambda=\pi / m+3 h\left(m=\sqrt{A_{1} B_{1}}\right)$, contient un zéro au moins de la fonction $y(x)$ (resp. $z(x)$ ).

Enfin, en supposant que l'on a

$$
A(x)>0, \quad B(x)<0, \quad \Delta A(x)>0, \quad \Delta B(x)<0 \quad \text { pour } x \in J,
$$

on obtient quelques inégalités pour les expressions

$$
\sqrt{|B|}|y|, \quad \sqrt{|A|}|z| \text { et }|\Delta y(x)|, \quad|\Delta z(x)| .
$$

Les propriétés analogues pour les solutions des équations différentielles sont bien connues.

Introduction. Dans cet article nous considérons le système d'équations aux différences finies

$$
\Delta y=h A(x) z, \quad \Delta z=h B(x) y,
$$

où les fonctions $A(x)$ et $B(x)$ sont continues dans l'intervalle $J$ et $h$ est une constante positive. Soit $y(x), z(x)$ une solution continue du système (I).

Nous appelons une solution $y(x), z(x)$ oscillante dans l'intervalle $\left(x_{0}, \infty\right)$, si les deux fonctions $y(x)$ et $z(x)$ changent de signe + et - une infinité de fois dans l'intervalle $\left(x_{0}, \infty\right)$.

Supposons remplies les inégalités

$$
0<A_{1} \leqslant A(x), \quad B(x) \leqslant-B_{1}<0, \quad x \in\left(x_{0}, \infty\right),
$$

où $A_{1}, B_{1}$ sont des constantes positives. 
Dans notre travail [2] nous avons obtenu, entre autres, les résultats suivants: $1^{\circ} \mathrm{Si}$ les inégalités (II) sont satisfaites, toute solution du système (I) est oscillante. Désignons par $x_{1}, x_{2}, \ldots, x_{n}, \ldots\left(x_{1}<x_{2}<\ldots<x_{n}<\ldots\right)$ et $\xi_{1}, \xi_{2}, \ldots, \xi_{n}, \ldots\left(\xi_{1}<\xi_{2}<\ldots<\xi_{n}<\ldots\right)$ les zéros consécutifs plus grands que $x_{0}$ des fonctions $y(x)$ et $z(x)$ respectivement. Soient remplies les inégalités

$$
x_{n+1}-x_{n}>h, \quad \xi_{n+1}-\xi_{n}>h \quad(n=1,2,3, \ldots) .
$$

Si les conditions (II) et (III) sont satisfaites les zéros des fonctions $y(x)$ et $z(x)$ se séparent alternativement et de plus on a $x_{n}<\xi_{n}<x_{n+1}$ $(n=1,2,3, \ldots)$ dans l'intervalle $\left\langle x_{0}, \infty\right)$.

$2^{\circ}$ Considérons les deux systèmes d'équations: (I) et

$$
\begin{aligned}
\Delta u & =h a(x) v \\
\Delta v & =h b(x) u
\end{aligned} \quad(h>0),
$$

où les coefficients $a(x)$ et $b(x)$ sont des fonctions continues dans l'intervalle $J$. Supposons remplies les inégalités

$$
0<a(x) \leqslant A(x), \quad B(x) \leqslant b(x)<0
$$

dans le sous-intervalle $(\alpha, \beta)$ de $J$.

THÉORÈme T (DE COMPARAISON). Supposons remplies les inégalités (V). Si de plus les conditions suivantes sont satisfaites:

$1^{\circ} \Delta A(x) \geqslant 0, \Delta a(x) \leqslant 0$ dans un intervalle $J$;

$2^{\circ} y(x)>0$ pour $x \epsilon\langle\alpha, \beta)$;

$3^{\circ}$ il existe une solution $u(x), v(x)$ de (IV) telle que $y(\alpha)=u(a)$, $z(a) \leqslant v(a) \leqslant 0$

$4^{\circ} n$ est un entier positif tel que $n h \leqslant \beta-\alpha<(n+1) h$. Dans oes conditions on $a y(a+i h) \leqslant u(a+i h)(i=1,2, \ldots, n)$.

Désignons par $a, b$ et $c_{1}, c$ les zéros consécutifs de la fonction $y(x)$ et $z(x)$ respectivement et, de plus, soit $x_{0}<c_{1}<a<c<b<\infty$.

Dans le $\S 1$ nous avons démontré que la longueur de tous les intervalles $\left(c_{1}, a\right),(a, c)$ et $(c, b)$ ne dépasse pas $(2+1 / p) h$, où $p=h^{2} A_{1} B_{1}>0$.

Ensuite ( $\$ 2$ et $\$ 3$ ) nous trouvons des conditions suffisantes pour que soient remplies les inégalités

$$
y(c+i h) \leqslant u(c+i h) \quad(i=0,1,2, \ldots, n ; n \geqslant 2),
$$

où $u(x)$ est une solution de l'équation différentielle du second ordre

$$
u^{\prime \prime}(x)+A_{1} B_{1} u(x)=0 .
$$

Nous avons obtenu aussi ( $\$ 3$ et $\S 4$ ) des conditions suffisantes pour que les longueurs des intervalles $(a, c)$ et $(c, b)$ ne surpassent pas $\frac{3}{2} h+$ $+\pi / 2 \sqrt{A_{1} B_{1}}$ pour $0<p \leqslant 2$. 
Enfin (\$5) nous avons obtenu quelques inégalités pour les différences $|\Delta y(x)|,|\Delta z(x)|$ et pour $|B|^{1 / 2}|y|, A^{1 / 2}|z|$ dans l'intervalle $(a, b)$.

M. Biernacki a considéré [1] des problèmes analogues dans le cas de l'équation aux différences finies: $\Delta^{2} y+h^{2} A(x) y=0$.

§ 1. Considerons le système d'équations aux différences finies

$$
\begin{aligned}
& \Delta y=h A(x) z \quad(h>0), \\
& \Delta z=h B(x) y \quad
\end{aligned}
$$

où les coefficients $A(x)$ et $B(x)$ sont des fonctions continues pour $x \epsilon J$. Nous supposons de plus que ces coefficients remplissent les inégalités suivantes:

$$
0<A_{1} \leqslant A(x), \quad B(x) \leqslant-B_{1}<0
$$

( $A_{1}$ et $B_{1}$ sont des constantes positives) pour $x \epsilon J$.

Soit $y(x), z(x)$ une solution continue et oscillante du système (1.1) dans l'intervalle $J$. Nous supposons que les zéros de ces fonctions se séparent alternativement. Désignons par $a, b$ et $c_{1}, c$ les zéros consécutifs de la fonction $y(x)$ et $z(x)$ respectivement, c'est-à-dire on a $z\left(c_{1}\right)=y(a)$ $=z(c)=y(b)=0$, où $c_{1}<a<c<b$.

Nous allons maintenant démontrer le

THÉORÈME I. Si les conditions (1.2) sont remplies, nous avons les inégalités

$$
a-c_{1}<P, \quad c-a<P, \quad b-c<P,
$$

ò̀ $\dot{P}=(2+1 / p) h$ et $p=h^{2} A_{1} B_{1}$ c'est-à-dire $b-a<2 P, c-c_{1}<2 P$.

En particulier si $h_{1}=\sqrt{2 / A_{1} B_{1}}$, on a $2 P \doteq 5 h_{1}$.

Démonstration.

$1^{\circ}$ Définissons l'entier positif $n$ par les inégalités

$$
n h \leqslant b-c<(n+1) h .
$$

$\Pi$ suffit de considérer le cas $y(x)>0$ pour $x \epsilon(a, b)$ Or, nous avons

$$
y(c+i h)>0 \quad(i=0,1,2, \ldots, n-1) .
$$

D'après (1.1) et (1.2) on obtient l'inégalité( $\left.{ }^{1}\right)$

$$
\Delta z(c+i h)=h(B y)_{c+i h}<0 \quad(i=0,1,2, \ldots, n-1) .
$$

Il vient donc

$$
z(c+n h)<z(c+(n-1) h)<\ldots<z(c+h)<0,
$$

d'où on déduit la relation

$$
|\Delta y(c+i h)|=h(A|z|)_{c+i h} \geqslant h A_{1}|z(c+h)| \quad(i=1,2, \ldots, n-1),
$$

(1) Nous posons: $\varphi_{\gamma} \equiv \varphi(\gamma)$. 
et par suite nous obtenons l'inégalité

$$
(n-1) h A_{1}|z(c+h)| \leqslant \sum_{i=1}^{n-1}|\Delta y(c+i h)| \leqslant y(c) .
$$

En posant dans la relation (1.5) $i=0$ et en tenant compte de l'hypothèse $B(x) \leqslant-B_{1}<0$ nous aurons

$$
h B_{1} y(c) \leqslant h(|B| y)_{c}=|z(c+h)| \text {. }
$$

Or, d'après (1.6) et (1.7) on a l'inégalité

$$
(n-1) p \leqslant 1
$$

et par conséquent

$$
n \leqslant 1+\frac{1}{p},
$$

d'où selon (1.3), on déduit l'inégalité

$$
b-c<\left(2+\frac{1}{p}\right) h .
$$

En particulier, si $h_{1}=\sqrt{2 / A_{1} B_{1}}$ on obtient l'inégalité

$$
b-c<\frac{5}{2} h_{1} .
$$

$2^{\circ}$ La démonstration de la deuxième partie du théorème I est tout analogue à celle de $1^{\circ}$. Définissons l'entier positif $r$ par les inégalités

$$
r h \leqslant c-a<(r+1) h .
$$

Nous avons $y(a)=0$ et $y(x)>0$ pour $x \epsilon(a, c>$ de plus on a $z(c)=0$. n vient done

$$
y(c-i h)>0 \quad(i=0,1,2, \ldots, r-1 ; r \geqslant 2) .
$$

D'après (1.1), (1.2) et (1.12) nous obtenons les inégalités

$$
\Delta z(c-i h)=h(B y)_{c-i h}<0 \quad(i=1,2, \ldots, r-1),
$$

d'où on déduit

$$
0<z(c-i h)<z(c-(i+1) h) \quad(i=1,2, \ldots, r-1) .
$$

De l'équation $\Delta y=h A(x) z$ nous obtenons les relations

$$
\Delta y(c-i h)=h(A z)_{c-i h} \quad(i=1,2, \ldots, r)
$$

et par suite d'après (1.13) on a

$$
\Delta y(c-i h) \geqslant h A_{1} z(c-h)>0 \quad(i=1,2, \ldots, r) .
$$


Nous posons dans (1.15) successivement $i=2,3, \ldots, r$ et faisons la somme des inégalités obtenues; il résulte donc

$$
y(c-h)-y(c-r h) \geqslant(r-1) h A_{1} z(c-h) .
$$

D'après l'hypothèse $B(x) \leqslant-B_{1}<0$ et en vertu de la relation

$$
\Delta z(c-h)=z(c)-z(c-h)=h(B y)_{c-h},
$$

nous obtenons l'inégalité

$$
z(c-h) \geqslant h B_{1} y(c-h) \text {. }
$$

En tenant compte des inégalités (1.12) et (1.17) on peut écrire l'inégalité (1.16) sous la forme suivante:

$$
y(c-h) \geqslant(r-1) h^{2} A_{1} B_{1} y(c-h),
$$

d'où on déduit l'inégalité

$$
r \leqslant 1+\frac{1}{p}
$$

et ensuite, d'après (1.11),

$$
c-a<\left(2+\frac{1}{p}\right) h
$$

Dans le cas particulier où $h_{1}=\sqrt{2 / A_{1} B_{1}}$ nous obtenons l'inégalité

$$
c-a<\frac{5}{2} h_{1} \text {. }
$$

$3^{\circ}$ Supposons maintenant que $c_{1}$ et $c$ sont deux zéros consécutifs de la fonction $z(x)$, c'est-à-dire $z\left(c_{1}\right)=z(c)=0\left(c_{1}<a<c<b\right)$. Il suffit de considérer le cas $z(x)>0$ pour $x \in\left(c_{1}, c\right)$.

En posant $y(x)=-\eta(x), z(x)=-\zeta(x)$ dans le système (1.1) nous obtenons le système suivant

$$
\begin{aligned}
& \Delta \eta=h A(x) \zeta(x) \\
& \Delta \zeta=h B(x) \eta(x) \quad(h>0) .
\end{aligned}
$$

Dans ce cas nous avons $\eta(a)=y(a)=0$ et $\eta(x)>0$ pour $x \epsilon\left(c_{1}, a\right)$, parce que l'inégalité $y(x)<0$ est remplie pour $x \epsilon\left(c_{1}, a\right)$; on a aussi $\zeta\left(c_{1}\right)$ $=z\left(c_{1}\right)=0$ et de plus $\zeta(x)<0$ pour $x \in\left(c_{1}, a\right)$, car l'inégalité $z(x)>0$ est satisfaite pour $x \epsilon\left(c_{1}, a\right)$. En appliquant pour le système (1.18) un raisonnement analogue à celui employé dans le cas $1^{\circ}$ on obtient l'inégalité

$$
a-c_{1}<\left(2+\frac{1}{p}\right) h .
$$

Le théorème I est done démontré. 
§ 2. Considérons un système (1.1)

$$
\begin{aligned}
& \Delta y=h A(x) z \\
& \Delta z=h B(x) y
\end{aligned} \quad(h>0)
$$

et supposons que les inégalités (1.2) sont remplies. Soit $y(x), z(x)$ une solution du système (1.1). Nous supposons dans la suite que chacune des fonctions $y(x)$ et $z(x)$ est continue et qu'entre deux zéros consécutifs de $y(x)$ il y a un seul zéro de $z(x)$, entre deux zéros consécutifs de $z(x)$ un seul zéro de $y(x)$.

Nous allons maintenant comparer la fonction $y(x)$ et une solution $u(x)$ de l'équation différentielle du second ordre

$$
u^{\prime \prime}(x)+m^{2} u(x)=0 \quad\left(m=\sqrt{A_{1} B_{1}}\right) .
$$

Supposons dans la suite que l'on a $p=h^{2} \cdot A_{1} B_{1} \leqslant 2$. Soit $y(a)=y(b)$ $=0$ et $y(x)>0$ pour $x \epsilon(a, b)$. Il existe un point $x=c(a<c<b)$ où $z(c)=0$.

D'après (1.1) et (1.3) on obtient $\left({ }^{2}\right) y(c)=y(c+h)>0$. De la condition $p \leqslant 2$ on déduit qu'il existe une solution $u(x)$ de l'équation (2.1) qui satisfait aux conditions

$$
u(c)=y(c), \quad u(c+h)=y(c+h) .
$$

En effet, cette solution est de la forme suivante

$$
u(x)=\frac{y(c)}{\cos \frac{m h}{2}} \cos \left[m\left(x-c-\frac{h}{2}\right)\right]
$$

où l'on a les inégalités $0<\frac{1}{2} m h \leqslant \sqrt{2} / 2<\pi / 4$.

La distance entre deux zéros consécutifs de $u(x)$ est égale à $\pi / m$, cependant d'après notre hypothèse on $a k \sqrt{2} / m$. Il résulte donc que la solution considérée $u(x)$ ne s'annule pas dans l'intervalle $(c, c+h)$. Nous allons maintenant remplacer l'équation différentielle (2.1) par un système d'équations aux différences finies. En effet, d'après la formule d'interpolation de Lagrange nous avons

$$
\Delta^{2} u(x)=h^{2} u^{\prime \prime}(\xi), \quad \xi \epsilon(x, x+2 h),
$$

par conséquent nous pouvons écrire l'équation (2.1) sous la forme suivante

$$
\Delta^{2} u(x)+m^{2} h^{2} \frac{u(\xi)}{u(x)} u(x)=0
$$

(2) Cf. [2], p. 130. 
et nous la remplaçons par le système d'équations aux différences finies

$$
\begin{aligned}
& \Delta u(x)=h A_{1} v(x) . \quad(h>0) \\
& \Delta v(x)=h b_{1}(x) u(x) \quad
\end{aligned}
$$

où nous avons posé $b_{1}(x) \equiv-B_{1} \frac{u(\xi)}{u(x)}$.

Soient $\gamma$ et $\delta$ les zéros consécutifs de la fonction $u(x)$ et de plus soit $\gamma<c<c+h<\delta$. La fonction $b_{1}(x)$ est continue dans l'intervalle $(\gamma, \delta)$. Il est evident que $u(x)>0$ et $u(x+h)>0$ dans l'intervalle $(\gamma, \delta-h)$.

Nous allons maintenant démontrer le

LEMME 1. Si $0<p \leqslant 2$, l'inégalité $-B_{1}<b_{1}(x)<0$ est remplie pour $x \epsilon(c, \delta-h)$ et $\xi \epsilon(x, x+2 h)$.

Démonstration. La constante $B_{1}$ est positive et $u(x)>0, u(x+h)$ $>0$ pour $x \epsilon(c, \delta-h)$. Il suffit donc de démontrer que $u(\xi)>0$ pour $x \epsilon(c, \delta-h)$ et $\xi \epsilon(x ; x+2 h)$. En effet, selon (2.4) on obtient l'équation

$$
\Delta v(x)=-h B_{1} u(\xi), \quad \xi \epsilon(x, x+2 h) .
$$

D'après (2.5) il suffit de démontrer que l'on a $\Delta v(x)<0$ pour $\boldsymbol{x}$ $\epsilon(c, \delta-h)$. De la formule (2.3) il vient donc

$$
u(x+h)=\frac{y(c)}{\cos \left(\frac{1}{2} m h\right)} \cos \left[m\left(x-c+\frac{h}{2}\right)\right],
$$

d'où il résulte que l'on a

$$
\Delta u(x)=-2 y(c) \operatorname{tg}\left(\frac{1}{2} m h\right) \sin [m(x-c)], \quad x \epsilon(c, \delta-h) .
$$

Or, selon (2.4) nous obtenons

$$
v(x)=-\frac{2 y(c)}{h A_{1}} \operatorname{tg}\left(\frac{1}{2} m h\right) \sin [m(x-c)], \quad x \epsilon(c, \delta-h)
$$

et ensuite

$$
v(x+h)=-\frac{2 y(c)}{h A_{1}} \operatorname{tg}\left(\frac{1}{2} m h\right) \sin [m(x+h-c)]
$$

d'où on obtient

$$
\Delta v(x)=-\frac{4}{h A_{1}} \sin ^{2}\left(\frac{1}{2} m h\right) u(x+h) .
$$

On voit immédiatement de la formule (2.7) que l'inégalité $\Delta v(x)<0$ est remplie dans l'intervalle $c<x<\delta-h$, car $u(x+h)>0$ pour $x \in(c, \delta-h)$. La fonction $b_{1}(x)$ est done négative dans l'intervalle $(c, \delta-h)$.

Dans ce qui suit nous allons démontrer que l'on a

$$
-B_{1}<b_{1}(x)<0, \quad x \in(c, \delta-h) .
$$

6 - Annales Polonicl Mathematici Xoxrx.3 
En effet, nous avons

$$
b_{1}(x)=\frac{\Delta v(x)}{h u(x)}, \quad x \epsilon(c, \delta-h)
$$

et selon la relation (2.7) il vient

$$
b_{1}(x)=-\frac{4}{h^{2}} \operatorname{Ain}^{2}\left(\frac{1}{2} m h\right) \cdot \frac{u(x+h)}{u(x)} .
$$

Si $p=h^{2} A_{1} B_{1} \leqslant 2$, c'est-à-dire si $0<\frac{1}{2} m h \leqslant \frac{1}{2} \sqrt{2}<\pi / 4$, on obtient l'inégalité

et par conséquent

$$
0<\sin \left(\frac{1}{2} m h\right)<\frac{1}{2} m h
$$

$$
\sin ^{2}\left(\frac{1}{2} m h\right)<\frac{1}{4} h^{2} A_{1} B_{1} .
$$

D'après la formule (2.6) on a $v(c)=0$ et ensuite il résulte de (2.7) que $v(x)<0$ dans l'intervalle $(c, \delta-h)$. En tenant compte de $(2.4)$ nous obtenons donc

$$
\Delta u(x)=h A_{1} v(x)<0, \quad x \epsilon(c, \delta-h)
$$

c'est-à-dire

$$
u(x+h)<u(x), \quad x \epsilon(c, \delta-h),
$$

d'où on déduit que l'on a

$$
0<\frac{u(x+h)}{u(x)}<1, \quad x \epsilon(c, \delta-h) .
$$

En tenant compte de (2.10) et (2.11) dans (2.9) nous obtenons les inégalités

$$
-B_{1}=-\frac{4}{h^{2} A_{1}} \cdot \frac{1}{4} h^{2} A_{1} B_{1}<b_{1}(x)<0
$$

c'est-à-dire

$$
\left.B(x) \leqslant-B_{1}<b_{1}(x)<0, \quad x \epsilon<c, \delta-h\right) .
$$

Le lemme 1 est donc démontré.

\$ 3. Considérons maintenant les deux systèmes d'équations aux différences finies

et

$$
\begin{aligned}
& \Delta y(x)=h A(x) z(x) \quad(h>0) \\
& \Delta z(x)=h B(x) y(x)
\end{aligned}
$$

$$
\begin{aligned}
& \Delta u(x)=h A_{1} v(x) \\
& \Delta v(x)=h b_{1}(x) u(x) \quad(h>0),
\end{aligned}
$$


où

$$
b_{1}(x) \equiv-B_{1} \frac{u(\xi)}{u(x)}, \quad x \epsilon(a, b), \quad \xi \epsilon(x, x+2 h)
$$

D'après les conditions initiales (2.2) on obtient la relation $\Delta y(c)$ $=\Delta u(c)$ et par conséquent, en vertu de (1.1) et (2.4), on a $A(c) z(c)=A_{1} v(c)$. Lorsque $z(c)=0$ il vient

$$
z(c)=v(c)=0 \text {. }
$$

Selon le lemme 1 et l'hypothèse (1.2) nous avons

$$
B(x) \leqslant-B_{1}<b_{1}(x)<0, \quad x \in\langle c, \delta-h) .
$$

On peut appliquer aux deux systèmes (1.1) et (2.4) le théorème $T$ (de comparaison) $\left({ }^{3}\right)$.

En effet, le système d'équations (1.1) est le même que (I) et le système (2.4) est aussi identique à (IV) si l'on pose $a(x) \equiv A_{1}, b(x) \equiv b_{1}(x)$. Dans ce cas $\Delta a(x) \equiv 0$. On peut écrire les hypothèses (V) sous la forme suivante

$$
0<A_{1} \leqslant A(x), \quad B(x)<b_{1}(x)<0 .
$$

Ensuite nous remplaçons dans le théorème $\mathrm{T} \alpha$ par $c, \beta$ par $b$. Nous pouvons done énoncer le théorème de comparaison suivant pour les systèmes (1.1) et (2.4):

THÉORÈmE II. Supposons remplies les conditions suivantes: $1^{\circ} 0<A_{1}$ $\leqslant A(x), B(x) \leqslant-B_{1}<0$ et $\Delta A(x) \geqslant 0$ dans l'intervalle $J, 2^{\circ} y>0$ pour $c \leqslant x<b, 3^{\circ} n$ est un entier positif tel que $n h \leqslant b-c<(n+1) h$, $4^{\circ}$ soient encore remplies les conditions $y(c)=u(c), y(c+h)=u(c+h), z(c)=0$. Dans ces conditions on a les inégalités $y(c+i h) \leqslant u(c+i h)(i=1,2, \ldots, n)$.

Nous allons maintenant démontrer que l'on a

$$
b-c<\frac{\pi}{2 \sqrt{A_{1} B_{1}}}+\frac{3}{2} h .
$$

En effet, la distance entre les zéros consécutifs $\gamma, \delta$ d'une solution $u(x)$ de l'équation différentielle

$$
u^{\prime \prime}(x)+m^{2} u(x)=0 \quad\left(m=\sqrt{A_{1} B_{1}}\right),
$$

égale $\delta-\gamma=\pi / m$. Nous avons considéré plus haut la solution $u(x)$ de l'équation (2.1) donnée sous la forme

$$
u(x)=\frac{y(c)}{\cos \left(\frac{1}{2} m h\right)} \cos \left[m\left(x-c-\frac{h}{2}\right)\right]
$$

pour $0<p \leqslant 2$, c'est-à-dire $0<\frac{1}{2} m h \leqslant \sqrt{2} / 2<\pi / 4$.

( $\left.{ }^{3}\right)$ Voir l'introduction. 
D'après le théorème II on a $u(c+i h) \geqslant 0(i=0,1,2, \ldots, n)$ et de la formule (2.3) il résulte

$$
\underset{\gamma<x<\delta}{\max } u(x)=u\left(c+\frac{1}{2} h\right)>0,
$$

par conséquente nous obtenons l'inégalité

$$
\delta=c+\frac{1}{2} h+\frac{\pi}{2 \sqrt{A_{1} B_{1}}} \geqslant c+n h,
$$

c'est-à-dire $n h \leqslant \frac{\pi}{2 \sqrt{A_{1} B_{1}}}+\frac{1}{2} h$. Selon l'hypothèse $3^{\circ}$ du théorème II nous avons $b-c<(n+1) h$. Nous obtenons donc l'inégalité

$$
b-c<\frac{\pi}{2 \sqrt{A_{1} B_{1}}}+\frac{3}{2} h .
$$

Dans lé cas particulier où $h=\sqrt{\frac{2}{A_{1} B_{1}}}$ l'inégalité (3.3) se réduit à la suivante

$$
b-c<\frac{3 \sqrt{2}+\pi}{2} \cdot \frac{1}{\sqrt{A_{1} B_{1}}} .
$$

Dans ce $\S$ nous avons obtenu une limitation de $b-c$.

\$ 4. Nous allons maintenant chercher une limite supérieure de $c-a$. Le raisonnement est tout pareil à celui employé dans les $§ 2$ et $\S 3$.

Soit donné un système d'équations aux différences finies

$$
\begin{gathered}
\Delta y(x)=h A(x) z(x), \\
\Delta z(x)=h B(x) y(x),
\end{gathered}
$$

où les coefficients $A(x)$ et $B(x)$ satisfont aux conditions

$$
0<A_{1} \leqslant A(x), \quad B(x) \leqslant-B_{1}<0 \quad(x \in J) .
$$

Considérons une solution continue et oscillante $y(x), z(x)$ du système (1.1) et supposons que l'on a: $y(a)=y(b)=0$ et $y(x)>0$ pour $x \epsilon(a, b)$. Il existe un seul point $x=c(a<c<b)$ tel que $z(c)=0$. Par conséquent, d'après (1.1), on obtient $z(x)>0$ pour $x \epsilon\langle a, c)$ et $z(a)=z(a+h)>0$; de plus, selon (1.11), nous avons les inégalités

$$
z(a+i h)>0 \quad \text { pour }(i=2,3, \ldots, r-1), z(a+r h) \geqslant 0 .
$$

En supposant que $0<p \leqslant 2$ on peut comparer la fonction $z(x)$ et une solution $v_{1}(x)$ de l'équation différentielle

$$
v_{1}^{\prime \prime}(x)+m^{2} v_{1}(x)=0 \quad\left(m=\sqrt{A_{1} B_{1}}\right)
$$


qui satisfait aux conditions

$$
v_{1}(a)=z(a), \quad v_{1}(a+h)=z(a+h) .
$$

Cette solution prend la forme

$$
v_{1}(x)=\frac{z(a)}{\cos \frac{m h}{2}} \cos \left[m\left(x-a-\frac{h}{2}\right)\right]
$$

où $0<\frac{1}{2} m h \leqslant \sqrt{2} / 2<\pi / 4$. On peut remplacer l'équation (2.1 $\left.\alpha\right)$ par le système d'équations aux différences finies:

$$
\begin{aligned}
& \Delta v_{1}(x)=h B_{1} w(x), \\
& \Delta w(x)=h a_{1}(x) v_{1}(x), \quad x \in\langle a, c),
\end{aligned}
$$

où nous avons posé

$$
a_{1}(x) \equiv-A_{1} \frac{v_{1}\left(\xi_{1}\right)}{v_{1}(x)} \quad \operatorname{pour} x \epsilon\langle a, c), \xi_{1} \epsilon(x, x+2 h)
$$

En effet, selon la formule d'interpolation de Lagrange on a $\Delta^{2} v_{1}(x)$ $=h^{2} v_{1}^{\prime \prime}\left(\xi_{1}\right), \xi_{1} \epsilon(x, x+2 h)$ et par conséquent d'après $(2.1 \alpha)$ il vient

$$
h^{-2} \Delta^{2} v_{1}(x)+m^{2} \frac{v_{1}\left(\xi_{1}\right)}{v_{1}(x)} v_{1}(x)=0, \quad x \epsilon\langle a, c) .
$$

On voit immédiatement que l'équation (4.5) et le système (4.3) sont équivalents.

En posant $\eta(x)=-y(x)$ dans le système (1.1) nous obtenons

$$
\begin{aligned}
& \Delta z(x)=-h B(x) \eta(x), \\
& \Delta \eta(x)=-h A(x) z(x) .
\end{aligned}
$$

Dans le cas considéré la solution $z(x), \eta(x)$ du système (4.6) dans l'intervalle $\langle a, c\rangle$ et la solution $y(x), z(x)$ du système (1.1) dans l'intervalle $\langle c, b\rangle$ possedent les mêmes propriétés. $\Pi$ en est de le même de la solution $v_{1}(x), w(x)$ du système (4.3) dans l'intervalle $\langle a, c)$ et $u(x), v(x)$ du système (2.4) dans l'intervalle $\langle c, b\rangle$. Par conséquent le théorème II dans le cas des systèmes (4.6) et (4.3) peut être énoncé comme il suit:

THEORÈmE III. Supposons satisfaites les conditions suivantos: $1^{\circ} 0<A_{1}$ $\leqslant A(x), B(x) \leqslant-B_{1}<0$ et $\Delta B(x) \leqslant 0$ dans l'intervalle $J, 2^{\circ} y(x)>0$ pour $a<x \leqslant c, 3^{\circ}$ soit $r$ l'entier positif tel que $r h \leqslant c-a<(r+1) h, 4^{\circ}$ les conditions suivantes sont remplies: $z(a)=v_{1}(a), z(a+h)=v_{1}(a+h), y(a)$ $=0$. Dans ces hypothèses on $a$ les inégalités $z(a+i h) \leqslant v_{1}(a+i h)$ $(i=2,3, \ldots, r)$. 
Dans ce qui suit nous allons indiquer une application du théorème III. Nous démontrerons qu'il existe une limitation de la longueur de l'intervalle $(a, c)$, c'est-à-dire que l'on a l'inégalité

$$
c-a<\frac{\pi}{2 \sqrt{A_{1} B_{1}}}+\frac{3}{2} h .
$$

En effet, désignons par $\gamma_{1} \delta_{1}\left(\gamma_{1}<a<a+h<\delta_{1}\right)$ les zéros consécutifs de la solution $v_{1}(x)$, donnée par la formule (4.2). On sait que l'on a

$$
\delta_{1}-\gamma_{1}=\frac{\pi}{\sqrt{A_{1} B_{1}}} .
$$

De la formule (4.2) il vient donc

$$
\delta_{1}=a+\frac{1}{2} h+\frac{\pi}{2 \sqrt{A_{1} B_{1}}} .
$$

D'après le théorème III on obtient

et par conséquent

$$
0 \leqslant z(a+r h) \leqslant v_{1}(a+r h)
$$

$$
a+r h \leqslant \delta_{1} .
$$

En substituant (4.8) dans (4.9) nous avons l'inégalité

$$
r h \leqslant \frac{1}{2} h+\frac{\pi}{2 \sqrt{A_{1} B_{1}}} .
$$

Selon l'hypothèse $3^{\circ}$ du théorème III et en vertu de (4.10) nous obtenons l'inégalité

$$
c-a<\frac{\pi}{2 \sqrt{A_{1} B_{1}}}+\frac{3}{2} h
$$

c'est-à-dire l'inégalité (4.7).

En particulier, si $h=\sqrt{\frac{2}{A_{1} B_{1}}}$ l'inégalité (4.7) prend la forme

$$
c-a<\frac{3 \sqrt{2}+\pi}{2} \cdot \frac{1}{\sqrt{A_{1} B_{1}}} .
$$

En tenant compte des inégalités (3.3) et (4.7) nous pouvons énoncer le

Corollame. Si les conditions (1.2) et $\Delta A(x) \geqslant 0, \Delta B(x) \leqslant 0 \quad(x \in I)$ sont satisfaites, toute sous-intervalle de $J$ dont la longueur dépasse

$$
\lambda=\frac{\pi}{\sqrt{A_{1} B_{1}}}+3 h
$$


contient un zéro au moins de la fonction $y(x)$ ou $z(x)$, c'est-à-dire nous avons les inégalités

$$
b-a<\lambda, \quad c-c_{1}<\lambda .
$$

Dans le cas particulier où $h=\sqrt{\frac{2}{A_{1} B_{1}}}$ on a $\lambda=\frac{3 \sqrt{2}+\pi}{2 \sqrt{A_{1} B_{1}}}$.

\$ 5. Considérons le système d'équations aux différences finies

$$
\begin{aligned}
& \Delta y(x)=h A(x) z(x) \quad(h>0) . \\
& \Delta z(x)=h B(x) y(x)
\end{aligned}
$$

Supposons ensuite que l'on a

$$
A(x)>0, \quad B(x)<0, \quad \Delta A(x) \geqslant 0, \quad \Delta B(x) \leqslant 0
$$

dans l'intervalle $J$.

Soit une solution $y(x), z(x)$ du système (1.1) continue et oscillante dans l'intervalle $\left\langle x_{0}, \infty\right)$. Nous désignons par $a, b$ et $c_{1}, c$ des zéros consécutifs des fonctions $y(x)$ et $z(x)$ respectivement; soit de plus

$$
x_{0}<c_{1}<a<c<b<\infty \text {. }
$$

Nous allons maintenant démontrer le

Thífonème IV. Supposons que les inégalités (5.1) et (5.2) sont remplies. Soient les entiers $r$ et $n$ définis par les inégalités $r h \leqslant c-a<(r+1) h$ et $n h \leqslant b-c<(n+1) h$. Soit $k$ l'un des nombres $1,2, \ldots, r$. Supposons qu'il existe un entier $s(1 \leqslant s \leqslant n)$ tel que l'on ait:

$$
|y(c+(s-1) h)| \geqslant|y(c-k h)| \geqslant|y(c+s h)| \text {. }
$$

Dans ces conditions nous avons l'inégalité

$$
A^{1 / 2}(c-k h)|z(c-k h)|<A^{1 / 2}(c+s h)|z(c+s h)|
$$

et a fortiori

$$
|\Delta y(c-k h)|<|\Delta y(c+s h)| .
$$

Démonstration. On peut facilement vérifier que l'on a

$$
\Delta\left(A z^{2}\right)=2 A z \Delta z+A(\Delta z)^{2}+(z+\Delta z)^{2} \Delta A
$$

et

$$
\Delta\left(B y^{2}\right)=2 B y \Delta y+B(\Delta y)^{2}+(y+\Delta y)^{2} \Delta B
$$

En faisant la différence de (5.3) et (5.4) nous obtenons

$$
\begin{aligned}
\Delta\left(A z^{2}\right)-\Delta\left(B y^{2}\right)=2(A z \Delta z-B y \Delta y)+A(\Delta z)^{2}-B(\Delta y)^{2}+ \\
+(z+\Delta z)^{2} \Delta A-(y+\Delta y)^{2} \Delta B
\end{aligned}
$$

6 - Annales Polonicl Mathematicl Xxrx.3 
D'après le système (1.1) on a l'égalité

$$
A z \Delta z-B y \Delta y=0
$$

et, par conséquent, de la relation (5.5) résulte l'inégalité

$$
\Delta\left(A z^{2}\right)>\Delta\left(B y^{2}\right)+(z+\Delta z)^{2} \Delta A-(y+\Delta y)^{2} \Delta B
$$

En posant dans l'inégalité (5.6) successivement

$$
x=c-k h, \quad x=c-(k-1) h, \ldots, x=c+(s-1) h
$$

et en faisant la somme des inégalités obtenues nous avons

$$
\begin{aligned}
\left(A z^{2}\right)_{c+8 h}-\left(A z^{2}\right)_{c-k h}> & \left(B y^{2}\right)_{c+s h}-\left(B y^{2}\right)_{c-k h}+ \\
& +\sum_{i=-k}^{s-1}\left[(z+\Delta z)^{2} \Delta A-(y+\Delta y)^{2} \Delta B\right]_{c+i h} .
\end{aligned}
$$

D'après (5.1) et en vertu de l'inégalité $y^{2}(c+s h) \leqslant y^{2}(c-k h)$ il vient:

$$
\left(B y^{2}\right)_{c+s h}-\left(B y^{2}\right)_{c-k h} \geqslant\left(B_{c+s h}-B_{c-k h}\right) y_{c+s h}^{2}=y_{c+s h}^{2} \sum_{i=-k}^{s-1} \Delta B(c+i h) \text {. }
$$

En profitant de (5.8) dans l'inégalité (5.7) on obtient a fortiori

$$
\begin{aligned}
\left(A z^{2}\right)_{c+s h}-\left(A z^{2}\right)_{c-k h}> & \sum_{i=-k}^{s-1}\left[(z+\Delta z)^{2} \Delta A\right]_{c+i h}+ \\
& +\sum_{i=-k}^{s-1}\left\{\left[y^{2}(c+s h)-(y+\Delta y)^{2}\right] \Delta B\right\}_{c+i h}
\end{aligned}
$$

Les deux suites

$$
\left\{(y+\Delta y)^{2}\right\}_{c-i h} \quad(i=1,2, \ldots, r)
$$

et

$$
\left\{(y+\Delta y)^{2}\right\}_{c+j h} \quad(j=0,1,2, \ldots, n-1)
$$

sont non croissantes. Par conséquent nous obtenons les inégalités

$$
y^{2}(c+s h) \leqslant(y+\Delta y)_{c+i h}^{2} \quad(i=-k, \ldots,-1,0,+1, \ldots, s-1) .
$$

Donc en tenant compte des inégalités (5.9) et (5.10) il vient:

$$
\left(A^{1 / 2}|z|\right)_{c-k h}<\left(A^{1 / 2}|z|\right)_{c+s h}
$$

et ensuite

c'est-à-dire

$$
h(A|z|)_{c-k h}<h(A|z|)_{c+s h}
$$

$$
|\Delta y(c-k h)|<|\Delta y(c+s h)| .
$$

Le théorème III est donc démontré. 
\$6. Nous avons obtenu plus haut quelques propriétés de la fonction $y(x)$. Dans ce qui suit nous allons obtenir des résultats analogues pour $z(x)$.

Nous démontrérons en effet le

THétèm V. Désignons par $a_{1}$ et $b_{1}$ des zéros consécutifs. de la fonction $z(x)\left(x_{0}<a_{1}<b_{1}<\infty\right)$ et soit $x=c_{1}\left(a_{1}<c_{1}<b_{1}\right)$ le seul point dans l'intervalle $\left(a_{1}, b_{1}\right)$ où l'on $a y\left(c_{1}\right)=0$. Soient définis les entiers $r_{1}$ et $n_{1}$ par les inégalités: $r_{1} h \leqslant c_{1}-a_{1}<\left(r_{1}+1\right) h, n_{1} h \leqslant b_{1}-c_{1}<\left(n_{1}+1\right) h$. Soit ensuite $k_{1}$ l'un des nombres $1,2, \ldots, r_{1}$ et supposons qu'il existe un entier $s_{1}\left(1 \leqslant s_{1} \leqslant n_{1}\right)$ tel que l'an ait:

$$
\left|z\left(c_{1}+\left(s_{1}-1\right) h\right)\right| \geqslant\left|z\left(c_{1}-k_{1} h\right)\right| \geqslant\left|z\left(c_{1}+s_{1} h\right)\right| \text {. }
$$

Dans ces hypothèses nous avons l'inégalité

$$
\left|B\left(c_{1}-k_{1} h\right)\right|_{1}^{1 / 2}\left|y\left(c_{1}-k_{1} h\right)\right|<\left|B\left(c_{1}+s_{1} h\right)\right|^{1 / 2}\left|y\left(c_{1}+s_{1} h\right)\right|
$$

et a fortiori on obtient

$$
\left|\Delta z\left(c_{1}-k_{1} h\right)\right|<\left|\Delta z\left(o_{1}+s_{1} h\right)\right| .
$$

Démonstration. Le raisonnement est toute pareil à celui du théorème IV. L'inégalité (5.6) peut être écrite sous la forme

$$
-\Delta\left(B y^{2}\right)>-\Delta\left(A z^{2}\right)+(z+\Delta z)^{2} \Delta A-(y+\Delta y)^{2} \Delta B \text {. }
$$

Posons dans (6.1) successivement

$$
x=c_{1}-k_{1} h, \quad c_{1}-(k-1) h, \ldots, c_{1}+\left(s_{1}-1\right) h
$$

et faisons la somme des inégalités obtenues; on a done

$$
\begin{aligned}
\left(B y^{2}\right)_{c_{1}-k_{1} h}-\left(B y^{2}\right)_{c_{1}+s_{1} h}> & \left(A z^{2}\right)_{c_{1}-k_{1} h}-\left(A z^{2}\right)_{c_{1}+s_{1} h}+ \\
& +\sum_{i=-k_{1}}^{s_{1}-1}\left[(z+\Delta z)^{2} \cdot \Delta A-(y+\Delta y)^{2} \Delta B\right]_{x=c_{1}+i h}
\end{aligned}
$$

et par conséquent

$$
\left(B y^{2}\right)_{c_{1}-k_{1} h}-\left(B y^{2}\right)_{c_{1}+s_{1} h}>\sum_{i=-k_{1}}^{s_{1}-1}\left\{\left[(z+\Delta z)^{2}-z^{2}\left(c_{1}+s_{1} h\right)\right] \Delta A\right\}_{x-c_{1}+i h}
$$

D'après les équations (1.1) il vient

$$
z^{2}\left(c_{1}+s_{1} h\right) \leqslant(z+\Delta z)_{c_{1}+i h}^{2} \quad\left(i=-k_{1}, \ldots,-1,0,1, \ldots, s_{1}-1\right) .
$$

En tenant compte des inégalités (6.3) et de l'hypothèse $\Delta A \geqslant 0$, nous obtenons d'après (6.2) l'inégalité suivante:

$$
\left|B\left(c_{1}-k_{1} h\right)\right|^{1 / 2}\left|y\left(c_{1}-k_{1} h\right)\right|<\left|B\left(c_{1}+s_{1} h\right)\right|^{1 / 2}\left|y\left(c_{1}+s_{1} h\right)\right|
$$


Selon (5.1) nous avons $B<0, \Delta B \leqslant 0$ et, par conséquent, en vertu de (6.4) nous obtenons a fortiori l'inégalité

$$
h\left|B\left(c_{1}-k_{1} h\right)\right|\left|y\left(c_{1}-k_{1} h\right)\right|<h\left|B\left(c_{1}+s_{1} h\right)\right|\left|y\left(c_{1}+s_{1} h\right)\right| .
$$

Des équations (1.1) il résulte immédiatement que l'inégalité (6.5) peut être écrite sous la forme suivante:

$$
\left|\Delta z\left(c_{1}-k_{1} h\right)\right|<\left|\Delta z\left(c_{1}+s_{1} h\right)\right| .
$$

Le théorème $\nabla$ est donc démontré.

\section{Travaux cités}

[1] M. Biernaoki, Sur l'équation $h^{-2} \Delta^{2} y(x)+A(x) y(x)=0$, Prace Mat.-Fiz. 47 (1949), p. 49-53.

[2] Z. Butlewski, Théorème de comparaison pour un système d'équations aux differences finies, Comm. Math. 14 (1970), p. 127-136. 